Jarjanazi, Hamdi

\section{Trinucleotide repeat expansions in familial/sporadic breast/ovarian cancer: searching for novel predisposition genes}

Hamdi Jarjanazi ${ }^{1}$, Nalan Gokgoz ${ }^{1}$, Irene L. Andrulis ${ }^{1-4} \&$ Hilmi Ozcelik ${ }^{1,2}$

${ }^{1}$ Dept. of Pathology and Laboratory Medicine, Center for Cancer Genetics, Samuel Lunenfeld Research Institute, Mount Sinai Hospital, Ontario, Canada

${ }^{2}$ Dept. of Laboratory Medicine and Pathobiology, Faculty of Medicine, University of Toronto, Toronto, Ontario, Canada.

${ }^{3}$ Dept. of Molecular and Medical Genetics, University of Toronto, Toronto,

Ontario, Canada.

${ }^{4}$ Ontario Cancer Genetics Network (OCGN)

The inheritance of mutated version of either $B R C A 1$ or $B R C A 2$ confers a high risk of developing breast cancer. However, only $10-20 \%$ of the breast cancer families carries BRCA mutations, suggesting the presence of other predisposition genes associated with high risk of breast cancer. In our ongoing breast cancer study, we have observed a trend of a decrease of the age of onset from one generation to the next in some breast cancer families. This suggested the potential presence of clinical anticipation, which has been shown to associate with trinucleotide repeat expansions in many diseases. This study aims at identifying putative breast cancer predisposition gene(s), which have undergone intergenerational expansions of trinucleotide repeats during germ line transmission using the Repeat Expansion Detection (RED) technique. DNA from mothers and daughters of breast cancer families has been screened for the presence of trinucleotide expansions. We have started the screening for expansions in (CAG) and (CGG) repeats initially since they are more commonly shown to be associated with anticipation in genetic disorders. Besides using molecular genome scan, with increasing human genome data, we have been using genome databases for identifying candidate trinucleotide repeat containing genes to study their potential involvement with breast cancer. Our approach has the potential to allow the rapid identification of novel breast cancer predisposition genes which will provide obvious benefits for families with breast cancer, as well as the potential for insights into the pathobiology of this disease.
Petros, John A.

\section{Classification of adult renal epithelial neoplasms by gene expression profiling using CDNA microarrays}

John Petros ${ }^{1-3}$, Andrew Young ${ }^{1}$, Mahul Amin ${ }^{1}$, So-Dug Lim ${ }^{1}$, Fray Marshall ${ }^{1,2}$, James Madara ${ }^{1} \&$ Andrew Neish ${ }^{1}$

${ }^{1}$ Emory University Depts Urology, Pathology

${ }^{2}$ Winship Cancer Institute

${ }^{3}$ Atlanta V A Medical Center

We analyzed expression of 7,075 genes in four conventional renal cell carcinomas (RCC), one chromophobe RCC, and two oncocytomas using cDNA microarrays. Expression profiles were compared between tumors using hierarchical clustering algorithms. Tumors segregated into two major gene expression classes correlated with histopathologic diagnoses; specifically, conventional RCCs were clearly distinguished from chromophobe RCC/oncocytomas. Tumors were classified with high resolution using a selected panel of 70 differentially expressed genes. Chromophobe carcinoma and oncocytomas overexpressed strikingly similar genes, including several related to oxidative phosphorylation and several expressed normally by the distal nephron, consistent with the mitochondrionrich morphology of these tumors and the theory that both lesions are related histogenetically to distal nephron epithelium. Conventional RCCs underexpressed mitochondrial and distal nephron genes, and were further distinguished from chromophobe RCC/oncocytomas by overexpresion of vimentin and class II MHC-related molecules. Expression patterns of four genes - vimentin, CD74, parvalbumin, and galectin-3 - were validated in additional tumors (34) by immunohistochemistry. Vimentin was a sensitive, specific marker for conventional RCCs, and parvalbumin was a promising marker for chromophobe RCC/oncocytomas. In conclusion, gene expression profiling identified distinct expression patterns related to the pathobiology of renal neoplasms and was an effective approach for discovering immunomarkers for renal tumor subtypes. 